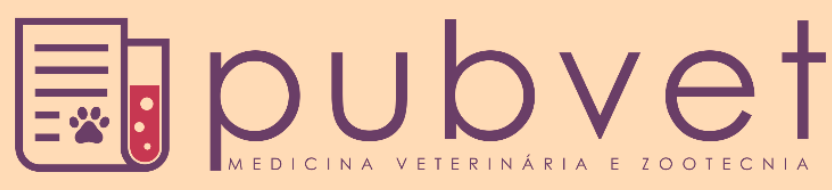

https://doi.org/10.31533/pubvet.v15n01a731.1-6

\title{
Quérion dermatofítico em cadela: Relato de caso
}

\section{Vitor Lima Torres ${ }^{1 \mathfrak{G}}$, Paulo Victor Silva Moraes ${ }^{1 \mathfrak{9}}$, Victor Machado de Carvalho ${ }^{1 \mathfrak{G}}$, Alexandre Tavares Camelo Oliveira ${ }^{10}$, Rodrigo Fonseca de Medeiros Guedes ${ }^{2} \boldsymbol{\theta}$, Adriana de Queiroz Pinheiro $^{30}$, Tiago Cunha Ferreira ${ }^{3 * 90}$}

\author{
'Aluno da Faculdade de Veterinária da Universidade Estadual do Ceará (UECE). Fortaleza - CE, Brasil. \\ ${ }^{2}$ Professor da Faculdade de Veterinária da Universidade de Fortaleza (UNIFOR). Fortaleza - CE, Brasil. \\ ${ }^{3}$ Professor (a) da Faculdade de Veterinária da Universidade Estadual do Ceará (UECE). Fortaleza - CE, Brasil. \\ *Autor para correspondência, E-mail: tiago.cunha@uece.br
}

Resumo. A dermatofitose é uma afecção fúngica que pode acometer cães e gatos de diversas idades. Uma apresentação pouco comum para esta doença é sua forma nodular, chamada quérion. Diversas técnicas são indicadas na literatura para o diagnóstico, dentre elas destaca-se a citologia, por ser uma técnica de simples execução, pouco dispendiosa e rápida. Neste relato, uma fêmea canina foi atendida apresentando um aumento de volume em região de pálpebra superior direita. Ao exame físico dermatológico, a lesão encontravase alopécica e inflamada, com presença de conteúdo viscoso à palpação, sendo compatível com quérion dermatofítico. Foi realizado exame citológico com punção por agulha fina, bem como cultura fúngica da secreção proveniente da lesão. A avaliação citológica revelou infiltrado inflamatório e estruturas compatíveis com artroconídeos fúngicos. Após 30 dias da semeadura ocorreu crescimento de colônias de Microsporum canis. A terapia da paciente baseou-se no uso de Itraconazol em suspensão $(10 \mathrm{mg} / \mathrm{kg} / \mathrm{dia})$ por um período de 45 dias. Ao final do tratamento obteve-se cura clínica, com ausência de processo inflamatório e total repilação do local lesionado.

Palavras-chave: Dermatofitose, lesões nodulares, citologia cutânea

\section{Dermatophytic kerion in the female dog: Case report}

Abstract. Dermatophytosis is a fungal disease that can affect dogs and cats of different ages. An unusual presentation for this disease is the nodular form, called kerion. Several techniques are indicated in the literature for diagnosis, among them cytology stands out because it is a simple, inexpensive, and fast technique. In this report, a canine female was treated with an increase in volume in the upper right eyelid region. Upon physical dermatological examination, the lesion was alopecic and inflamed, with the presence of viscous content on palpation, being compatible with dermatophyte cherion. The cytological examination was performed with a fine-needle puncture, as well as the fungal culture of the secretion from the lesion. The cytological evaluation revealed inflammatory infiltrate and structures compatible with dermatophyte arthroconidia. After 30 days of sowing, the Microsporum Canis colonies grew. The patient's therapy was based on the use of Itraconazole in suspension $(10 \mathrm{mg} / \mathrm{kg} / \mathrm{day})$ for 45 days. At the end of the treatment, a clinical cure was obtained, with no inflammation and total repilation of the injured site.

Keywords: Dermatophytosis, nodular lesions, cutaneous cytology

\section{Querion dermatofitico en hembra canina: Reporte de un caso}

Resumen: La dermatofitosis es una afección fúngica que puede afectar a perros y gatos de diferentes edades. Una presentación inusual de esta enfermedad es su forma nodular, llamada Querion. En la literatura se indican varias técnicas para el diagnóstico, entre las 
cuales destaca la citología porque es una técnica simple, económica y rápida. En este informe, una hembra canina fue tratada con un aumento de volumen en la región del párpado superior derecho. Tras el examen físico dermatológico, la lesión era alopécica e inflamada, con presencia de contenido viscoso a la palpación, siendo compatible con el Querion dermatofito. El examen citológico se realizó con punción con aguja fina, así como cultivo fúngico de la secreción de la lesión. La evaluación citológica reveló infiltrado inflamatorio y estructuras compatibles con artroconidos dermatofitos. Después de 30 días de siembra, crecieron las colonias de Microsporum canis. La terapia de la paciente se basó en el uso de itraconazol en suspensión $(10 \mathrm{mg} / \mathrm{kg} /$ día $)$ durante un período de 45 días. Al final del tratamiento, se obtuvo la curación clínica, sin inflamación y repilación total del sitio lesionado.

Palabras clave: Dermatofitosis, lesiones nodulares, citología cutánea

\section{Introdução}

A dermatofitose é uma doença fúngica cutânea muito comum entre os animais de companhia, sendo causada, costumeiramente, por microrganismos dos gêneros Microsporum spp., Trichophyton spp. e Epidermophyton spp. Mais de 30 espécies já foram identificadas dentro de tais gêneros, as quais, devido à sua capacidade pleomórfica, tornam-se naturalmente contagiosas e potencialmente zoonóticas (Moriello et al., 2017; Weitzman \& Summerbell, 1995).

Estes fungos possuem tropismo por regiões queratinizadas, podendo atingir o estrato córneo de pelos, unhas e pele (Rhodes \& Werner, 2014). A doença consiste em uma micose superficial, mas que pode atingir camadas mais profundas, visto que a resposta inflamatória do local pode resultar em uma reação de hipersensibilidade capaz de gerar lesões nodulares tanto em cães quanto em gatos, estando os quérions entre os usualmente mais encontrados (Ferreira et al., 2006). Alguns achados estão comumente presentes em animais acometidos pela doença, como a presença de pápulas, crostas, eritema, hiperpigmentação, descamação da pele, alopecia e em alguns casos onicogrifose (Moriello et al., 2017).

O diagnóstico desta micose pode ser realizado por meio de avaliação direta de pelo, lâmpada de Wood, Real Time PCR, isolamento fúngico e citologia. Contudo, dependendo do padrão lesional, algumas dessas ferramentas pode não ser efetiva (Rhodes \& Werner, 2014). A citologia e o isolamento fúngico, são ferramentas capazes de reduzir o percentual de casos falso negativos. Porém, no caso do isolamento, uma desvantagem é que seus resultados podem demorar devido ao tempo de crescimento da cultura, variando de 10 a 21 dias (Moriello, 2014; Rezusta et al., 2016). De modo geral, o isolamento fúngico é facilitado em animais com manifestações clínicas bem evidentes. No entanto, a ausência de sintomatologia em alguns indivíduos não descarta a possibilidade de o fungo atingir os seres humanos (Ates et al., 2008).

Por tratar-se de uma abordagem clínica não usual da dermatofitose, o objetivo do presente trabalho é descrever um caso de quérion dermatofítico ocasionado por Microsporum canis em cão.

\section{Relato de caso}

Uma fêmea canina, 12 anos de idade, não castrada, foi atendida apresentando aumento de volume em região de pálpebra superior direita, com evolução de sete dias. Ao exame físico dermatológico, a lesão encontrava-se alopécica e inflamada, com presença de conteúdo viscoso à palpação, sendo compatível com quérion dermatofítico (Figura 2). Ressalta-se que a paciente não possuía outros padrões lesionais ao longo do corpo, sendo este o único local clinicamente acometido. Foi realizado teste da Lâmpada de Wood na região afetada, não havendo fluorescências dos pelos.

Em avaliação clínico-geral constatou-se que a paciente possuía vacinação antiviral e antirrábica atualizadas, não apresentava ectoparasitos em inspeção visual e era soronegativa para leishmaniose. Ao exame físico as mucosas apresentavam-se normocoradas, temperatura retal de $38,7^{\circ} \mathrm{C}$, a paciente estava normohidratada, e à palpação apresentou linfonodos poplíteos e submandibulares em tamanho normal.

$\mathrm{Na}$ ocasião, realizou-se exame citológico com punção por agulha fina para melhor elucidação do caso clínico, assim como cultura fúngica da secreção proveniente da lesão (Figura 1). Em avaliação 
citológica, foi possível visualizar um infiltrado inflamatório composto por neutrófilos, macrófagos e eosinófilos, assim como numerosas estruturas redondas ou ovoides, de citoplasma basofílico com fino halo periférico, sendo morfologicamente compatíveis com artroconídeos de dermatófitos. A cultura fúngica foi realizada em ágar Mycosel ${ }^{\circledR}$, com o objetivo de confirmar os achados citológicos, sendo o meio de cultura gentilmente cedido pelo Laboratório de Microbiologia da Faculdade de Veterinária da Universidade Estadual do Ceará. No ágar utilizado, foi observado, após 30 dias da semeadura, crescimento de colônias algodonosas compatíveis com as originadas de fungos dermatófitos. Para avaliação da espécie envolvida na lesão, procedeu-se com a coloração com lactofenol azul de algodão, sendo possível identificar os macroconídeos de M. canis.

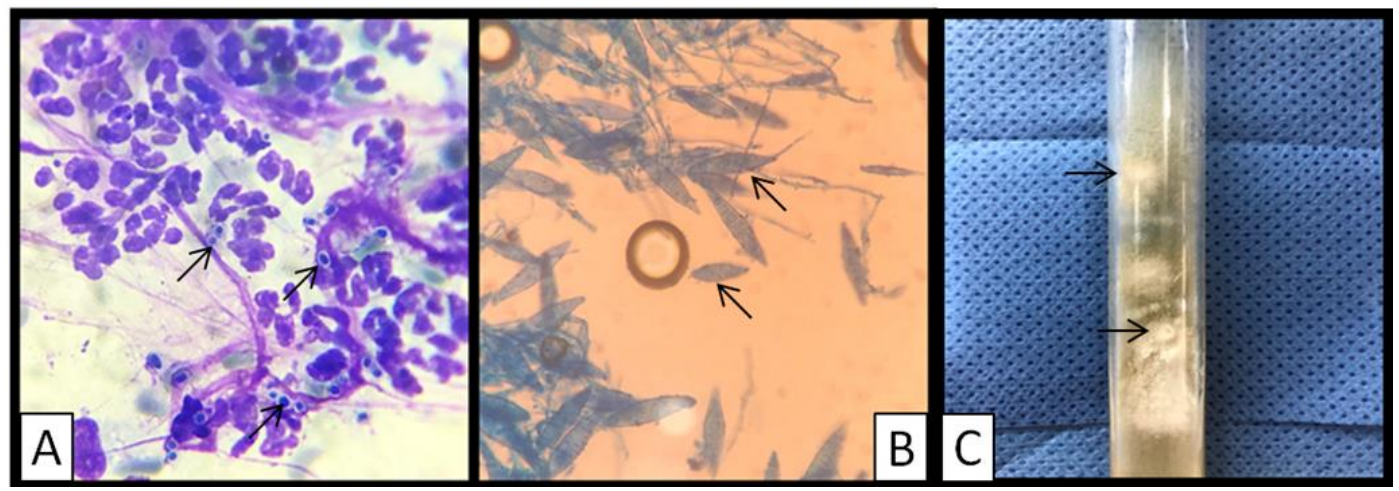

Figura 1. (A) Fotomicrografia de avaliação citológica, demonstrando processo inflamatório piogranulomatoso, entremeado por estruturas compatíveis com artroconídeos de dermatófitos (setas) corado por panótico rápido, aumento de 1000x; (B) microscopia de cultura fúngica de lesão em quérion dermatofítico, com visualização de macroconídeos de $M$. canis (setas); (C) macroscopia da cultura apresentando colônias algodonosas (setas), em meio Mycosel ${ }^{\circledR}$.

A terapia da paciente baseou-se no uso de Itraconazol em suspensão $(10 \mathrm{mg} / \mathrm{kg} / \mathrm{dia})$ por um período de 45 dias, sendo iniciado imediatamente após a identificação dos artroconídeos na citologia, visto que as colônias de dermatófito podem apresentar crescimento lento, como ocorreu no presente caso. Devido aos potenciais efeitos colaterais relacionados ao uso do itraconazol (Moriello et al., 2017), realizaramse ainda avaliações bioquímicas hepáticas seriadas por meio da dosagem de alanina aminotransferase (ALT) e aspartato aminotransferase (AST) séricas a cada 14 dias. Não foram observadas alterações em tais parâmetros até o término da terapia. Obteve-se cura clínica, com ausência de processo inflamatório e total repilação do local lesionado (Figura 3). Em situação posterior, realizou-se nova cultura fúngica com material proveniente do mesmo local que, anteriormente, apresentava-se alterado, não havendo crescimento de colônias no meio utilizado.

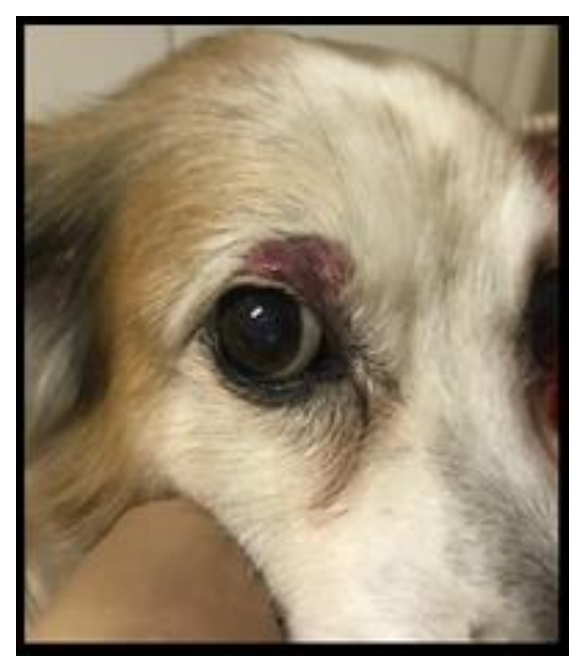

Figura 2. Lesão dermatológica de característica alopécica, eritematosa e nodular em região de pálpebra superior direita da paciente.

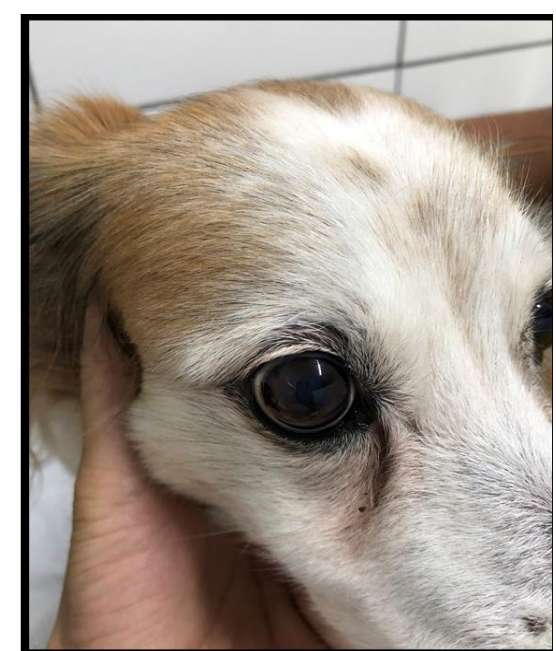

Figura 3. Aspecto dermatológico de região periocular após 45 dias de tratamento com Itraconazol, apresentando repilação de área afetada. 


\section{Discussão}

O caso descrito demonstra a apresentação clínica, diagnóstica e terapêutica de uma lesão nodular única, localizada em região periocular, que teve como causa base uma infecção dermatofítica. Nos caninos, geralmente as lesões causadas por dermatófitos variam de acordo com estado imunológico do animal ou com o grau de patogenicidade da cepa fúngica. Estas lesões normalmente são discretas e acompanhadas apenas por alopecia e descamação da região afetada. Por outro lado, em alguns casos mais extremos, a reação inflamatória pode ser grave, ocorrendo infecções bacterianas secundárias ou resultando em lesões nodulares (Cruz, 2010). Uma possível explicação para reação inflamatória grave em cães pode estar relacionada ao fato de dermatófitos ambientais serem agentes etiológicos ocasionais, não estando totalmente adaptado ao hospedeiro (Cruz, 2010; Miller et al., 2013). Em casos de lesões nodulares causadas por $M$. canis, como relatado neste estudo, pode-se hipotetizar que a cepa poderia ser mais patogênica ou ter sido inoculada diretamente no tecido subcutâneo.

Gross et al. (2005) relatam que este tipo de apresentação nodular é comumente associado a infecções por Microsporum gypseum ou Trichophyton mentagrophytes. Porém, neste caso, a espécie fúngica isolada foi $M$. canis, sendo compatível com dois levantamentos realizados por Cornegliani et al. (2009) e Albanese \& Caruso (2007), demonstrando que a infecção por $M$. canis foi a mais comum dentre os animais estudados.

Uma ferramenta que pode auxiliar no exame clínico em casos suspeitos de dermatofitose é o teste da Lâmpada de Wood (Cruz, 2010). Alguns microrganismos, como M. canis, ao metabolizarem o triptofano presente nos pelos, produzem fluorescência verde-amarelada nas lesões. Entretanto, este exame deve ser interpretado com cautela, devido à possibilidade de falsos positivo e negativo (Robert \& Pihet, 2008). No presente trabalho, o teste da Lâmpada de Wood foi negativo, corroborando com o estudo de Cornegliani et al. (2009), onde nenhum dos animais foi positivo no teste. Em lesões com padrão de quérion, espera-se que o resultado do teste seja negativo, visto que a lesão atinge camadas mais profundas da pele (Ferreira et al., 2006). Por outro lado, o levantamento feito por Albanese \& Caruso (2007) demonstrou que 38\% dos animais do estudo foram positivos. Isto pode ter ocorrido possivelmente devido a presença de pelos remanescentes na lesão.

A progressão da infecção por dermatófitos está associada a resposta imunológica modulada pelo hospedeiro, onde reações de hipersensibilidade tardia (mediada por células $\mathrm{T}$ ) resultam na maioria das manifestações clínicas do paciente (Jones, 1993). Nos casos em que o fungo alcança a derme ou promove uma reação de hipersensibilidade exacerbada, a reação inflamatória incitada pelo agente desencadeará um processo piogranulomatoso, o qual pode ser observado na citologia da lesão.

A forma clássica da dermatofitose ocorre normalmente em pacientes mais jovens devido a quantidade de pelos em fase de crescimento, o que oferta uma maior quantidade de substrato para o fungo (Rhodes \& Werner, 2014). Uma hipótese para a apresentação nodular da doença, deve-se ao fato de o animal adulto ter um sistema imunológico mais desenvolvido, reagindo de forma mais intensa à presença fungo (Gross et al., 2005).

A citologia corada por panótico é uma técnica de baixo custo, rápida e de simples execução que pode ser utilizada como ferramenta auxiliar no diagnóstico da dermatofitose (Robert \& Pihet, 2008). O diagnóstico presuntivo por exame citológico é uma das indicações relatadas no consenso mais recente publicado por Moriello et al. (2017) e mostrou-se bastante útil no caso em questão, onde foram visualizadas estruturas compatíveis com artroconídios fúngicos. Em outros trabalhos, esta técnica demonstrou ser bastante relevante, identificando precocemente o mesmo padrão citológico em $90 \%$ e 30\% de seus pacientes, respectivamente (Albanese \& Caruso, 2007; Cornegliani et al., 2009). A realização desse tipo de análise é fundamental, pois caso revele a presença de elementos fúngicos, o tratamento pode ser realizado antecipadamente, minimizando assim os danos ao animal. Vale ressaltar que mesmo com o diagnóstico presuntivo através da citologia, uma amostra da lesão deve ser colhida e enviada para o laboratório onde deverá ser realizada uma cultura, a fim de se confirmar o diagnóstico e descobrir o agente etiológico. Este procedimento foi realizado neste caso e após 30 dias o diagnóstico de dermatofitose foi confirmado.

O itraconazol é um derivado triazólico sintético de primeira geração que apresenta um largo espectro de ação nas micoses superficiais e sistêmicas em animais, sendo uma das principais opções para o 
tratamento da dermatofitose em cães e gatos (Pereira, 2009; Skidgel \& Erdös, 2006). Moriello et al. (2017) concluem no último consenso que o itraconazol e a terbinafina são os fármacos mais eficazes e seguros para o tratamento da dermatofitose.

Em um estudo foi relatada a eficácia fungicida do itraconazol contra vários agentes fúngicos, com destaque para sua eficácia contra M. canis e T. Mentagrophytes (Van Cutsem, 1989; Van Cutsem et al., 1987). Levando em conta que o itraconazol tem um amplo espectro de ação, ele foi o fármaco escolhido para o tratamento do caso, visto que a citologia não é capaz de revelar a espécie fúngica envolvida na infecção. A apresentação da droga em suspensão facilitou o cálculo da dose bem como a administração da mesma pelos proprietários. Houve uma boa resposta terapêutica, sem efeitos colaterais e o paciente obteve cura clínica ao final dos 45 dias de tratamento.

\section{Conclusão}

A partir do exposto, pode-se concluir que as lesões nodulares cutâneas podem estar associadas a infecções por dermatófitos, sendo recomendada a inclusão desta enfermidade tegumentar na lista de diagnósticos diferenciais para este padrão lesional. Além disso, ressalta-se a importância do exame citológico no diagnóstico da Dermatofitose, o qual fornece dados acerca do microrganismo e padrão inflamatório associados à lesão, agilizando possíveis intervenções terapêuticas.

\section{Referências}

Albanese, F., \& Caruso, C. (2007). Il kerion dermatofitico: aspetti eziologici, clinici, diagnostici e terapeutici in 39 cani. Veterinaria, 21(5), 9.

Ates, A., Ilkit, M., Ozdemir, R., \& Ozcan, K. (2008). Dermatophytes isolated from asymptomatic dogs in Adana, Turkey: A preliminary study. Journal de Mycologie Médicale, 18(3), 154-157. https://doi.org/10.1016/j.mycmed.2008.07.001

Cornegliani, L., Persico, P., \& Colombo, S. (2009). Canine nodular dermatophytosis (kerion): 23 cases. Veterinary Dermatology, 20(3), 185-190. https://doi.org/10.1111/j.1365-3164.2009.00749.x

Cruz, L. C. H. (2010). Micologia veterinária (Vol. 1). Revinter.

Ferreira, R. R., Machado, M. L. S., Spanamberg, A., \& Ferreiro, L. (2006). Querion causado por Microsporum gypseum em um cão. Acta Scientiae Veterinariae, 34(2), 179-182.

Gross, T. L., Ihrke, P. J., Walder, E. J., \& Affolter, V. K. (2005). Pustular and nodular diseases with adnexal destruction. Skin Diseases of the Dog and Cat: Clinical and Histopathologic Diagnosis: John Wiley \& Sons, 420-459.

Jones, H. E. (1993). Immune response and host resistance of humans to dermatophyte infection. Journal of the American Academy of Dermatology, 28(5), S12-S18. https://doi.org/10.1016/S01909622(09)80302-2

Miller, W. H., Griffin, C. E., Campbell, K. L., \& Muller, G. H. (2013). Muller and Kirk's Small Animal Dermatology. Elsevier Health Sciences.

Moriello, K. (2014). Feline dermatophytosis: aspects pertinent to disease management in single and multiple cat situations. Journal of Feline Medicine and Surgery, 16(5), 419-431.

Moriello, K. A., Coyner, K., Paterson, S., \& Mignon, B. (2017). Diagnosis and treatment of dermatophytosis in dogs and cats. Clinical Consensus Guidelines of the World Association for Veterinary Dermatology. Veterinary Dermatology, 28(3), 266--e68. https://doi.org/10.1111/vde.12806.

Pereira, S. A. (2009). Esporotricose felina: estudo terapêutico no Rio de Janeiro. Tese (Doutorado em Pesquisa Clínica em Doenças Infecciosas)-Instituto Nacional de Infectologia Evandro Chagas, Fundação Oswaldo Cruz, Rio de Janeiro.

Rezusta, A., de la Fuente, S., Gilaberte, Y., Vidal-García, M., Alcalá, L., López-Calleja, A., Ruiz, M. A., \& Revillo, M. J. (2016). Evaluation of incubation time for dermatophytes cultures. Mycoses, 59(7), 416-418. https://doi.org/10.1111/myc. 12484

Rhodes, K. H., \& Werner, A. H. (2014). Dermatologia de pequenos animais. Roca; $2^{\mathrm{a}}$ edição.

Robert, R., \& Pihet, M. (2008). Conventional Methods for the Diagnosis of Dermatophytosis. 
Mycopathologia, 166(5-6), 295-306. https://doi.org/10.1007/s11046-008-9106-3

Skidgel, R. A., \& Erdös, E. G. (2006). Goodman \& Gilman's The Pharmacological Basis of Therapeutics. McGraw-Hill, New York.

Van Cutsem, J. (1989). The In-vitro Antifungal Spectrum of Itraconazole. Mycoses, 32(s1), 7-13. https://doi.org/10.1111/j.1439-0507.1989.tb02290.x

Van Cutsem, J., Van Gerven, F., \& Janssen, P. A. J. (1987). Activity of Orally, Topically, and Parenterally Administered Itraconazole in the Treatment of Superficial and Deep Mycoses: Animal Models. Reviews of Infectious Diseases, 9(Supplement_1), S15-S32. https://doi.org/10.1093/clinids/9.Supplement_1.S15

Weitzman, I., \& Summerbell, R. C. (1995). The dermatophytes. Clinical Microbiology Reviews, 8(2), 240 LP - 259. https://doi.org/10.1128/CMR.8.2.240

Histórico do artigo:

Recebido: 5 de agosto de 2020.

Aprovado: 1 de setembro de 2020.

Disponível online: 26 de novembro de 2020.
Licenciamento: Este artigo é publicado na modalidade Acesso Aberto sob a licença Creative Commons Atribuição 4.0 (CC-BY 4.0), a qual permite uso irrestrito, distribuição, reprodução em qualquer meio, desde que o autor e a fonte sejam devidamente creditados. 